УДК: 616.69-008.14: 616.379-008.64

ЭРЕКТИЛЬНАЯ ДИСФУНКЦИЯ У БОЛЬНЫХ САХАРНЫМ ДИАБЕТОМ 1 ТИПА: МЕТОДЫ ДИАГНОСТИКИ И ЛЕЧЕНИЯ

\author{
Курбатов Д.Г., Дубский С.А., Лепетухин А.Е., Роживанов Р.В., Шварц Я.Г. \\ ФГБУ Эндокринологический Научный Центр Минздрава России, г.Москва \\ Адрес: 117036, г.Москва, ул.Дм.Ульянова, 11, тел. (499)3203687
}

Эл.почта: kurbatov.d@mail.ru

\begin{abstract}
В данном обзоре литературы освещены вопросы эпидемиологии, классификачия, патофизиология, а также диагностика и методы лечения эректильной дисфункции у больных сахарным диабетом 1 типа. Эректильная дисфункиия у молодых пациентов с сахарным диабетом представляет собой серьезную проблему по причине достаточно широкой распространенности, а также влияния на психологическое состояние больных и качество жизни в целом. Подчеркнуто, что своевременная диагностика с правильным определением формы эректильной дисфункции позволяет обоснованно и адекватно подобрать терапию для каждого конкретного пащиента.
\end{abstract}

Ключевые слова: сахарный диабет, эректильная дисфункция

\title{
ERECTILE DYSFUNCTION IN PATIENTS WITH TYPE 1 DIABETES: DIAGNOSIS AND TREATMENT METHODS
}

\author{
Kurbatov D.G., Dubskiy S.A., Lepetukhin A.E. Rozhivanov R.V., Schwartz J.G. \\ Endocrinology Research Center, Moscow
}

In this review of the literature shows the epidemiology, classification, pathophysiology, diagnosis and treatments for erectile dysfunction in patients with type 1 diabetes. Erectile dysfunction in young patients with diabetes mellitus is a serious problem because of fairly widespread, and the impact on the psychological state of patients and quality of life in general. Emphasized that timely diagnosis with the correct definition of a form of erectile dysfunction can reasonably and adequately pick therapy for each individual patient.

Key words: diabetes, erectile dysfunction

\section{ВВЕДЕНИЕ}

3 аболеваемость сахарным диабетом в мире стремительно увеличивается. По данным International Diabetes Federation на сегодняшний день более 371 млн. человек страдают сахарным диабетом (СД). Примерно 10\% от общего числа больных СД приходится на долю СД 1 типа [1].

Сексуальные расстройства, характеризующиеся снижением качества жиз- ни пациента, приводя к бесплодию и проблемам социального характера, наблюдаются более чем у 40\% больных СД $[2,3]$. Важно отметить, что половые расстройства у больных СД 1 типа дебютируют в более молодом возрасте по сравнению с популяцией без СД [4].

Превалирующим нарушением половой функции у больных СД является эректильная дисфункция (ЭД). В многочисленных исследованиях показано, 
что ЭД страдают до 35-55\% больных СД 1 типа [5], а риск ЭД у больных СД в 3 раза выше по сравнению с популяцией без СД [6].

Частота развития эректильных расстройств у больных СД зависит не только от возраста пациента, а также от длительности течения основного заболевания и продолжительности периода декомпенсации углеводного обмена $[7,8]$. На развитие ЭД влияет наличие сопутствующих заболеваний, осложнений СД и эффективность проводимой терапии [9]. Так, в нескольких исследованиях изучена связь наличия ЭД и поздних диабетических осложнений и показано, что ЭД выявлялась почти в 2 раза чаще у пациентов, имеющих диабетическую нефропатию или ретинопатию [10].

Диагностированная эректильная дисфункция у больных СД может быть косвенным признаком развития или прогрессирования атеросклеротического процесса и ишемической болезни сердца, а также являться первым проявлением диабетической нейропатии $[11,12]$. Подобное исследование, проведенное Роживановым Р.В. (2005) на базе ФГБУ «Эндокринологический Научный Центр» МЗ РФ, показывает сопоставимые с зарубежными данные по распространенности ЭД среди больных СД 1 и 2 типа, зависимости распространенности от возраста пациентов, длительности течения заболевания, а также взаимосвязи с уровнем компенсации углеводного обмена и наличием осложнений СД [13].

Учитывая распространенность ЭД при СД 1 типа, а также понимание того, что данное состояние не только снижает качество жизни молодых пациентов, но также может быть одним из симптомов таких осложнений СД, как диабетическая нейропатия, ИБС, атеросклероз, необходим своевременный, индивидуализированный и комплексный подход к диагностике и лечению ЭД у данной категории больных.

Классификаиия ЭД

Формы ЭД:

- Органическая (васкулогенная, нейрогенная, эндокринная)

- Психогенная

- Смешанная (органическая патология и психологический фактор)

- Медикаментозная

Функциональное состояние полового члена регулируется тонусом гладкой мускулатуры артериальных сосудов и трабекул кавернозных тел. После сексуальной стимуляции оксид азота (NO), синтезируемый эндотелием, увеличивает концентрацию гуанилатциклазы (ГМФ). Возрастающая концентрация циклического ГМФ (цГМФ) ведет к релаксации гладкомышечных волокон, усилению артериального притока и веноокклюзии в половом члене. Скорость распада цГМФ зависит от активности фермента 5-фосфодиэстеразы [14].

В основе развития ЭД при СД могут лежать одновременно несколько факто- 
ров (атеросклероз + нейропатия, нейропатия + психогенный фактор и др.)[15].

Эрекция полового члена регулируется различными изоформами NO-синтетазы нейронального, эндотелиального и гладкомышечного происхождения. [16]. Несколько биохимических механизмов объясняют возникновение эректильной дисфункции при СД. Сосудистый и нейрогенный компоненты вместе являются причинами ЭД при СД, так как известно, что эндотелиальная дисфункция приводит к развитию ишемической нейропатии, которая, в свою очередь, оказывает отрицательное действие на синтез NO. Во множестве исследований показано нарушение эндотелиально-зависимой и нейрогенной релаксации в кавернозных телах у больных СД с ЭД. Эта находка ассоциирована с недостаточностью NO. Более того, в некоторых зарубежных исследованиях показано значительное увеличение количества NO-синтетазосвязывающих участков в тканях кавернозных тел крыс через 2 месяца после индукции сахарного диабета [17]. Данный процесс аналогичен тем, что были выявлены в других сосудистых руслах, где эндотелий-зависимая релаксация сосудистой стенки была изменена в результате нарушения синтеза NO вследствие высоких концентраций глюкозы. Таким образом, дефект активности NOсинтетазы играет определенную роль в этиологии ЭД у больных СД, вследствие диффузной эндотелиальной дис- функции [18]. Было также показано, что релаксация гладкомышечных клеток в пещеристых телах у больных СД при электростимуляции была слабовыраженной из-за снижения продукции оксида азота NO-синтетазой. Важно отметить, что длительная гипергликемия индуцирует увеличение потребления никотинамидадениндинуклеотидфосфата (НАДФН), кофактора в продукции $\mathrm{NO}$, следовательно, снижая уровень оксида азота [19].

Чрезмерная генерация свободных радикалов ухудшает NO-индуцированную релаксацию и через накопление конечных продуктов гликирования (advanced glycation end-products (AGE)), циркулирующих в крови, которые также ответственны за развитие диабетических сосудистых осложнений [20].

AGE-продукты, аккумулируясь у больных СД, взаимодействуют со специфическими рецепторами тканей, которые подвергаются сосудистым поражениям, а также увеличивают экспрессию медиаторов сосудистого повреждения, высвобождение которых также стимулируется глюкозой. [21, 22, 23].

Все вышеперечисленные факторы вовлечены в патофизиологию кардиоваскулярных заболеваний, характеризующихся высокой смертностью (безболевая ишемия миокарда, внезапная сердечная смерть и др.), которые высоко ассоциированы с ЭД [24].

Нейропатия является важным составляющим в развитии диабетической 
ЭД. Было показано морфологическое повреждение автономных нервных волокон в тканях пещеристых тел у больных СД с ЭД [25]. Наличие периферической полинейропатии считается характерной для больных с ЭД, однако снижение скорости проведения нервного импульса по нервному волокну и вариабельность сердечного ритма все же регистрируются немного чаще у больных СД и ЭД, чем у больных с ЭД и полинейропатиями иного происхождения [26].

В многочисленных работах, посвященных патологическим изменениям нервной системы у больных СД, говорится о независимом первичном поражении периферических нервных волокон [27].

Автономная нейропатия представляется основным патогенетическим фактором ЭД у пациентов с СД. Пациенты с проявлениями периферической нейропатии чаще страдают ЭД, чем больные СД без полинейропатии [28]. Наиболее обоснованной метаболической гипотезой является теория полиолового обмена, согласно которой избыток глюкозы при СД метаболизируется по полиоловому типу, в конечном итоге, превращаясь в сорбитол и фруктозу, накопление которых в нервных клетках запускает развитие нейропатии [29]. Значимость гипергликемии в развитии диабетической нейропатии клинически подтверждается тем фактом, что при условии достижения компенсации уг- леводного обмена прогрессирование диабетической нейропатии снижается на 40-60\% [30].

Имеет значение и васкулогенная гипотеза развития нейропатии, основанная на обеднении эндоневрального кровотока, повышения эндоневрального сосудистого сопротивления и уменьшения оксигенации нерва. Согласно данной теории первичными являются патологические изменения эндоневральных сосудов и связанные с ними гипоксия и ишемия [31].

Все перечисленное говорит о важной роли периферической нейропатии в развитии ЭД у пациентов с СД. Многие авторы характеризуют это состояние как «нейрогенная ЭД», обращая тем самым внимание на ведущую роль диабетической нейропатии в эректильных расстройствах у таких больных [31, 32].

Вместе с васкулогенной и нейрогенной формами ЭД при СД распространена эндокринная ЭД, связанная с дефицитом андрогенов [33].

В недавно проведенных исследованиях продемонстрировано, что NOсинтетаза является андрогензависимым ферментом [34]. Об андрогенной зависимости NO-синтетазы свидетельствует то, что в нервных клетках тазовых парасимпатических ганглиев обнаружены рецепторы к андрогенам, в которых происходит синтез NO и вазоактивного интестинального пептида [35], а также стимуляция синтеза NO в ганглиях под влиянием андрогенов [36]. При этом 
гипогонадизм является частым симптомом у больных СД [37]. Причины андрогенного дефицита у мужчин с СД различны. Таковыми причинами могут быть избыточная масса тела или ожирение, а так же возрастное снижение секреции тестостерона. [38].

\section{Диагностика ЭД у больных СД}

Обследование пациента с ЭД при СД проводится по классической схеме, включающей сбор анамнестических данных, осмотр, а также лабораторные и инструментальные методы.

Важным моментом в сборе анамнеза у пациентов с СД является оценка течения основного заболевания, наличие или отсутствие осложнений СД, информация о принимаемых лекарственных препаратах.

При физикальном обследовании проводится измерение массы тела, роста и индекса массы тела, так как избыточный вес может быть причиной гипогонадизма. В некоторых случаях имеет смысл проводить дифференциальную оценку объема висцерального жира с помощью КТ-сканирования для более корректной оценки и прогнозирования рисков. Помимо этого оценивается состояние кожных покровов, характер и интенсивность оволосения, состояние мышечной системы и половых органов [39, 40].

При физикальном обследовании, с целью диагностики нейропатии, необходимо выполнение определенного минимума неврологических диагностиче- ских методик. Наиболее информативной является оценка кавернозного рефлекса [41]. Может быть рекомендована также оценка температурной, тактильной и вибрационной чувствительности полового члена [42].

Перечень специальных методов обследования при ЭД включает гормональное исследование крови, мониторирование ночных пенильных тумесценций, интракавернозное фармакодинамическое исследование, кавернозографию, ангиографию сосудов полового члена, ультразвуковую допплерографию сосудов полового члена и определение скорости распространения нервного импульса по n.pudendus [43].

Все вышеперечисленные методы обследования используются с целью диагностики ЭД любого генеза, однако наиболее информативным и единственно достоверным в настоящее время методом диагностики нейрогенной формы ЭД при СД является электронейромиография. К тестам, позволяющим оценить состояние сенсорных и эфферентных волокон, относятся промежностная электромиография латентного периода бульбокавернозного рефлекса, крестцовый скрытый тест, оценка вызванных дорсальных соматосенсорных потенциалов и исследование вибрационной перцептивной чувствительности. Для пациентов с СД и ЭД характерно отклонение результатов данных тестов от нормативных показателей. Например, для больных СД характерно увеличение 
латентного периода бульбокавернозного рефлекса [43]. Однако, вышеописанные тесты не дают представление о состоянии эфферентной автономной иннервации, ответственной за эрекцию полового члена. Исходя из вышеизложенного, при регистрации отклонения результатов тестов от нормы можно лишь предположить наличие автономной нейропатии в половом члене [44].

В качестве метода непосредственного исследования автономной кавернозной иннервации может проводиться регистрация электрической активности кавернозной гладкой мускулатуры при помощи интракавернозных или поверхностных накожных электродов. Данные, полученные при помощи этого метода, позволяют оценить состояние нервно-рефлекторной функции полового члена и выявить расстройства на уровне взаимодействия кавернозных тел и нервных окончаний. При исследовании автономной кавернозной иннервации у пациентов с сахарным диабетом регистрируются нерегулярные потенциалы с низкой амплитудой и медленной скоростью деполяризации, а также характерна десинхронизация парадоксальное повышение активности кавернозной ткани в ответ на введение вазоактивного препарата, в то время как у здоровых пациентов после интракавернозного введения вазоактивных препаратов потенциалы действия отсутствуют [45]. В настоящее время недостаточно данных относительно спе- цифичности и чувствительности данного метода.

Исходя из вышесказанного становится понятно, что диагностика собственно нейрогенной формы ЭД у больных СД представляет собой сложную задачу, особенно учитывая, что на сегодняшний день не существует высокочувствительного и специфичного метода диагностики. Следует учитывать то, что нарушение эрекции нередко бывает первым симптомом развивающейся нейропатии. Предположить нейрогенную форму ЭД у пациента с СД можно при наличии других проявлений диабетической нейропатии (снижение температурной, вибрационной и болевой чувствительности, различные проявления кардиоваскулярной и гастроинтестинальной форм автономной диабетической нейропатии, нераспознаваемая гипогликемия). Отсутствие данных за наличие сосудистой недостаточности и гипогонадизма наряду с жалобами на эректильные расстройства также могут свидетельствовать о нейрогенной ЭД.

\section{Лечение ЭД у больных СД}

При выборе метода лечения ЭД каждый пациент требует индивидульного подхода [45]. Учитывая возможность наличия у больных СД специфических осложнений, выбор методов лечения ЭД должен быть обоснованным [46]. Как известно, в настоящее время предпочтительно использование медикаментозных методов лечения ЭД, но что еще более важно: для эффективной те- 
рапии ЭД необходимо достижение стойкой компенсации углеводного обмена.

На сегодняшний день существует ряд методов местного лечения ЭД: вакуумная терапия, интракавернозная и трансуретральная фармакотерапия. Все эти методы имеют определенные недостатки, которые ограничивают их применение у больных СД, так как связаны с возможной травматизацией мягких тканей при интракавернозной фармакотерапии и слизистой оболочки уретры при трансуретральной фармакотерапии, что нежелательно для пациентов с СД ввиду высокого риска инфицирования микротравм [45].

В данный момент препаратами выбора для лечения ЭД являются ингибиторы фосфодиэстеразы 5 типа (силденафил, варденафил, тадалафил, уденафил). Препараты этой группы являются модуляторами эрекции, которые селективно ингибируют фермент ФДЭ-5, не оказывая прямого воздействия на гладкомышечные клетки полового члена, но усиливая действие NO, синтезирующегося в ответ на сексуальную стимуляцию. Таким образом, усиливаются физиологические процессы, отвечающие за возникновение и поддержание эрекции в ответ на сексуальную стимуляцию [45].

Многолетний опыт применения силденафила у больных с СД продемонстрировал его высокую эффективность в лечении ЭД [46, 47]. Об этом свиде- тельствуют долгосрочные исследования, результаты которых говорят о возможности длительного, безопасного, эффективного применения препарата без увеличения его дозы [48].

Эффективность варденафила в лечении ЭД у больных СД была изучена в многоцентровом, двойном слепом, плацебоконтролируемом исследовании, включившем 452 пациента. По данным оценки результатов исследования через 12 недель применения улучшение эрекции отмечалось у 52\% и 72\% мужчин, получавших 10 и 20 мг варденафила соответственно, тогда как в группе плацебо улучшение эрекции наблюдалось только у 13\% больных [49].

Эффективность и безопасность тадалафила у мужчин, включая больных СД, была изучена в исследовании Fonseca V. и соавт. (2006), в котором проводился мета-анализ данных двенадцати плацебо-контролируемых исследований у больных с ЭД, СД и без такового. В исследования были включены 1681 мужчина без СД и 637 мужчин с СД 1 и 2 типа, которые получали тадалафил, в дозах 10 и 20 мг либо плацебо в течение 12 недель. Пациенты с СД имели более выраженную ЭД, по сравнению с пациентами без СД, при этом балл ЭД по МИЭФ обратно коррелировал с уровнем НbA1c. По сравнению с плацебо тадалафил в дозах 10 и 20 мг достоверно улучшил эректильную функцию в обеих группах, что сопровождалось повышением качества жизни 
пациентов. При этом эффективность тадалафила не зависела от степени компенсации углеводного обмена и получаемой по поводу СД терапии. Таким образом, несмотря на более тяжелую ЭД у пациентов с СД тадалафил был эффективен и хорошо переносился [50]. Тадалафил имеет длительный период полувыведения - 17,5 часов, что обеспечивает достоверно большую длительность его действия, возвращая естественность в сексуальные отношения [51]. У пациента появляется возможность вести естественную сексуальную жизнь, что крайне актуально при наличии дополнительных психогенных факторов, отягощающих течение эректильной дисфункции у больных СД [52].

По данным исследователей у 20-40\% пациентов с ЭД терапия ингибиторами ФДЭ-5 оказывается неэффективной, что в некоторых случаях связано с наличием у пациентов андрогенного дефицита. Таким образом, в ряде случаев, представляется целесообразным назначение комбинированной терапии андрогенами и препаратами ингибиторов ФДЭ-5 с момента постановки диагноза пациентам, обладающим вышеописанными клиническими особенностями, что повышает эффективность терапии до $93 \%[53,54,55]$.

Применение препаратов ингибиторов ФДЭ-5 при терапии нарушений половой функции у больных сахарным диабетом может иметь и дополнительное преимущество в виде уменьшения симптомов генитальной нейропатии [49].

Так, в исследовании, включившем 16 мужчин с СД типа 1 и ЭД в возрасте 27 [25;29] лет с парестезиями в области ствола полового члена и нарушением чувствительности головки, получающих на протяжении 3 месяцев ингибитор ФДЭ-5, было отмечено не только полное устранение ЭД у всех пациентов (балл ЭД на фоне терапии 21 [21;22], $\mathrm{p}<0,001)$ и нормализация спонтанных эрекций, но и статистически значимое уменьшение жалоб, характерных для генитальной нейропатии, а так же улучшение температурной чувствительности полового члена [49].

Нейропротекторное действие ингибиторов ФДЭ-5 было отмечено при диабетической нейропатии. В публикации G. Hackett описаны 5 клинических случаев, где пациенты с болевой формой дистальной диабетической нейропатии отмечали значительное уменьшение тяжести симптоматики после регулярного приема ингибиторов ФДЭ-5. Положительное влияние ингибиторов ФДЭ-5 можно объяснить их воздействием на эндотелиальную функцию путем улучшения кровотока по vasa nervorum [56].

До настоящего времени наиболее перспективными в отношении лечения болевой формы диабетической полинейропатии являлись препараты альфалипоевой (тиоктовой) кислоты [57]. В ряде зарубежных и 1 отечественном 
не плацебо контролируемом исследовании показана эффективность данных препаратов в отношении лечения как диабетической полинейропатии в целом, так и нейрогенной ЭД в частности. Однако, следует учитывать, что эффект имел краткосрочный характер и определялся временем внутривенных инфузий [58].

Несмотря на достаточно широкий выбор средств для консервативной терапии ЭД у пациентов с СД 1 типа, существует группа больных, у которых данные методы лечения остаются неэффективными. В таком случае пациентам показано хирургическое лечение фаллоэндопротезирование.

\section{ЗАКЛЮЧЕНИЕ}

В данном обзоре литературы освещены вопросы эпидемиологии, классификация, патофизиология, а также диагностика и методы лечения ЭД у больных СД 1 типа. ЭД у молодых пациентов с СД представляет собой серьезную проблему по причине достаточно широкой распространенности, а также влияния на психологическое состояние больных и качество жизни в целом.

Из всего вышеизложенного становится ясно, что на сегодняшний день в арсенале врача имеется широкий спектр различных методов диагностики ЭД, однако до сих пор не разработано вы- сокоспецифного и чувствительного метода диагностики именно нейрогенной формы ЭД. Важно учитывать, что своевременная диагностика с правильным определением формы ЭД позволяет обоснованно и адекватно подобрать терапию для каждого конкретного пациента.

Лечение ЭД у больных СД 1 типа должно быть комплексным и направленным не только на улучшение собственно эректильной функции, а также на устранение патогенетических факторов развития ЭД, таких как хроническая гипергликемия, дислипидемия, андрогенный дефицит. В настоящее время предпочтение отдается медикаментозным методам лечения, лидирующее место среди которых занимают препараты из группы ингибиторов ФДЭ-5 типа благодаря высокой эффективности, безопасности и удобству в применении для пациентов. Стоит отметить, что препараты данной группы обладают нейропротективным действием, что особо важно для пациентов именно с нейрогенной формой ЭД, однако данный вопрос требует дальнейшего углубленного изучения.

Таким образом, несмотря на большие достижения в разработке методов диагностики и лечения ЭД, по прежнему остается много нерешенных вопросов, требующих дальнейших исследований. 


\section{ЛИТЕРАТУРА}

1. International Diabetes Federation [Internet]. Cited 2013 Dec 9. Url.: http://www.idf.org/worlddiabetesday/tool kit/gp/facts-figures.

2. Prevalence of erectile dysfunction and its correlates: a population-based study in Morocco / S. Berrada, N. Kadri, S. Mechakra-Tahiri, C. Nejjari // Int J Impot Res. - 2003. - Vol.15, Suppl 1. P.3-7.

3. Prevalence and correlates of erectile dysfunction in a population-based study in Belgium / R. Mak, G. De Backer, M. Kornitzer, J.M. De Meyer // Eur Urol 2002. - Vol.41(2). - P.132-138.

4. Роживанов, Р.В. Эректильная дисфункция у больных сахарным диабетом по данным контрольно-эпидемиологических исследований

Р.В. Роживанов, Ю.И. Сунцов, Д.Г. Курбатов // Сахарный диабет. 2009. - №2. - C.51-54.

5. Bancroft, J. Erectile dysfunction in men with and -without diabetes mellitus: a comparative study / J. Bancroft, P. Gutierrez // Diabet Med. - 1996. - Vol.13 (1). - P.84-89.

6. Schiel, R. Prevalence of sexual disorders in a selection-free diabetic population (JEVIN) / R. Schiel, U.A. Müller // Diabetes Res Clin Pract. - 1999, May. Vol.44(2). - P.115-121.

7. Vinik, A. Erectile dysfunction in diabetes. / A. Vinik, D. Richardson // Diabetes Rev. - 1998. - Vol.6(1). - P.1633.
8. Sexual function in men with diabetes type 2: association with glycemic control / J.H. Romeo, A.D. Seftel, Z.T. Madhun, D.C. Aron // J Urol. 2000. - Vol.163(3). - P.788-791.

9. Effect of chronic diseases on incidence of erectile dysfunction / R. Shiri, J. Koskimaki, M. Hakama et al. // Urology. - 2003. - Vol.62(6). - P.1097-1102.

10. Seyoum, B. Impotence in Ethiopian diabetic men / B. Seyoum // East. Afr. Med. J. - 1998. - Vol.75(4). P.208-210.

11. Comorbidities associated with diabetic foot complications among Asian Americans in southern California / P.Y. Han, R. Ezquerro, K.M. Pan et al. // J Am Podiatr Med Assoc. - 2003. Vol.93(1). - P.37-41.

12. Diabetic autonomic neuropathy / A.I. Vinik, R.E. Maser, B.D. Mitchell, R. Freeman // Diabetes Care. - 2003. Vol.26(5). - P.1553-1579.

13. Роживанов, Р.В. Эректильная дисфункция у больных сахарным диабетом: скрининг, структура, прогностическое значение: Автореф. дис. канд. мед. наук. - 2005.

14. Nitric oxide as a mediator of relaxation of the corpus cavernosum in response to nonadrenergic, noncholinergic neurotransmission / J. Rajfer, W.J. Aronson, P.A. Bush et al. // N Engl J Med. 1992. - Vol.326(2). - P.90-94.

15. Nusbaum, M.R. Erectile dysfunction: prevalence, etiology, and major risk 
factors / M.R. Nusbaum // J Am Osteopath Assoc. - 2002. - Vol.102(12), Sup.4. - P.1-6.

16. Protein and gene expression of nitric oxide synthase isoforms I and III in the rat penile shaft / C.M. Gonzalez, R.E. Brannigan, T. Bervig et al. // J Androl. - 2001. - Vol.22. - P.54-61.

17. Sullivan, M.E. Vascular risk factors and erectile dysfunction / M.E. Sullivan, S.R. Keoghane, M.A. // Br J Ural Int. - 2001. - Vol.87. - P.838-845.

18. Nitricoxide and penile erection, is erectile dysfunction another manifestation of vascular disease? / M.E. Sullivan, C.S. Thompson, M.R. Dashwood et al. // Cardiovasc Res. - 1999. - Vol.43(3). P.658-665.

19. Cartledge, J.J., Impairment of corpus cavernosal smooth muscle relaxation by grycosylated haemoglobin / J.J. Cartledge, I. Eardley, J.F.B. Morrison // Br J Urot Int. - 2001. - Vol.85. - P.735-741.

20. Cartledge, J.J. Advanced glycation end-products are responsible for the impairment of corpus cavemosal smooth muscle relaxation seen in diabetes / J.J. Cartledge, I. Eardley, J.F. Morrison // Br J Urol Int. - 2001. - Vol.87(4). P.402-407.

21. Molecular and biological properties of the vascular endothelial growth factor family of proteins / N. Ferrara, K. Houck, L. Jakeman, D.W. Leung // Endocr Rev. - 1992. - Vol.13(1). - P.18-32.

22. Advanced glycation end products induce expression of vascular endothelial growth factor by retinal Muller cells / C. Hirata, K. Nakano, N. Nakamura et al. // Biochem Biophys Res Commun. 1997. - Vol.236(3). - P.712-715.

23. Sarman, B. Role of endothelin-1 in diabetes mellitus / B. Sarman, M. Toth, A. Somogyi // Diabetes Metab Rev 1998. - Vol.14(2). - P.171-175.

24. Retter, A.S. Carnitine and its role in cardiovascular disease. / A.S. Retter // Heart Dis. - 1999. - Vol.1(12). P.108113.

25. Changes in the VIPergic, cholinergic and adrenergic innervation of human penile tissue in diabetic and non-diabetic impotent male / J. Lincoln, R. Crowe, P.F. Blacklay et al. // J Urol. - 1987. Vol.137(5). - P.1053-1059.

26. Neuropathy is a major contributing factor to diabetic erectile dysfunction / M.J. Hecht, B. Neundorfer, F. Kiesewetter F, M.J. Hilz // Neural Res. - 2001. Vol.23(6). - P.651-654.

27. Harati, Y. Diabetes and the nervous system / Y. Harati // Endocrinol Metab Clin North Am. - 1996. - Vol.25(2). - P.325-359.

28. Aetiopathogenesis and management of impotence in diabetic males: four years experience from a combined clinic / A. Veves, L. Webster, T.F. Chen et al. // Diabet Med. - 1995. - Vol.12(1). - P.77-82.

29. Hakim, L.S., Goldshtein I. Diabetic sexual dysfunction / L.S. Hakim, I. Goldshtein // Endocrinol. Metab. Clin. N. Am. - 1996. - Vol.25(2) - P.379-400. 
30. Stevens, M.J. Diabetic peripheral neuropathy. Current therapy of diabetes mellitus / M.J. Stevens, E.L. Feldman, D.A. Greene // Eds. R. A. Defronzo. - St. Louis: Mosby. - 1998. - P.160-165.

31. Балаболкин, М.И. Патогенез ангиопатий при сахарном диабете / М.И. Балаболкин, Е.М. Клебанова, B.М. Креминская // Сахарный диабет. - 1999. - №1. - C.2-9.

32. Калинченко, С.Ю. Нейрогенные нарушения половой функции у мужчин, страдающих сахарным диабетом / С.Ю. Калинченко, Р.В. Роживанов // Врач. - 2006. - №1 . - С.48-51.

33. Курбатов, Д.Г. Эректильная дисфункция у больных сахарным диабетом / Д.Г. Курбатов, Р.В. Роживанов, Д.В. Приймак // Русский медицинский журнал - 2009. - №17(25). C.1672-1676.

34. Rossi, P. Comparison between plasma concentrations of testosterone, nitric oxide and endothelin 1-2 in penile and brachial venous blood: preliminary results in men with psychogenic impotence / P. Rossi, F. Menchini Fabris, I. Fiorini et al. // Biomed. Pharmacother. - 1998. - Vol.52(7-8). - P.308-310.

35. Schirar, A. Localization of androgen receptor in nitric oxide synthase and vasoactive intestinal peptide containing neurons of the major pelvic ganglion innervating the rat penis / A. Schirar, C. Chang, J.P. Rousseau // J. Neuroendocrinol. - 1997. - Vol.9(2). P.141-150.
36. Hormones and nocturnal penile tumescence in healthy aging men / R.C. Schiavi, D. White, J. Mandeli, P. Schreiner-Engel // Arch. Sex. Behav. 1993. - Vol.22(3). - P.207-215.

37. Erectile dysfunction and lower androgenicity in type 1 diabetic patients / O. Alexopoulou, J. Jamart, D. Maiter et al. // Diabetes Metab. - 2001. Vol.27(3). - P.329-336.

38. Cunningham, M.J. Leptin's actions on the reproductive axis: perspectives and mechanisms / M.J. Cunningham, D.K. Clifton, R.A. Steiner // Biol. Reprod. - 1999. - Vol.60. - P.216-222.

39. Лоран, О.Б. Современные методы диагностики и лечения эректильных дисфункций / О.Б. Лоран, П.А. Щеплев, С.Н. Нестеров, С.А. Кухаркин// Русский медицинский журнал. - 2000. №8(3). - C.130-134.

40. Дедов, И.И. Федеральная целевая программа «Сахарный диабет». / И.И. Дедов, М.В. Шестакова, М.А. Максимова //Методические рекомендации. 2002.

41. Тиктинский, О.Л. Андрология. / О.Л. Тиктинский, В.В. Михайличенко // Медиа Пресс. - 1999.

42. Физикальное неврологическое обследование как скрининг-метод диагностики нейрогенной эректильной дисфункции у больных сахарным диабетом / Р.В. Роживанов, О.Н. Бондаренко, О.В. Удовиченко и др. // Врач. - 2005. - №5. - C.29-30. 
43. Нарушение половой функции у мужчин при сахарном диабете. / Под ред. М.И. Когана // Москва. - 2005.

44. Мазо, Е.Б. Сравнительная оценка показателей электромиографии полового члена и данных микроскопии кавернозной ткани у больных с эректильной дисфукцией в диагностике кавернозной иннервации / Е.Б. Мазо, Д.Г. Дмитриев, Д.Ю. Худолей // Андрология и генитальная хирургия. 2000. - №1. C.55-56.

45. Aggour, A. Evaluation of the role of corpus cavernosum electromyography as a noninvasive diagnostic tool in male erectile dysfunction / A. Aggour, $\mathrm{H}$. Mostafa, H. El-Shawaf // Int Urol Nephrol. - 1998. - №30(1). - C.75-79.

46. Ультразвуковая диагностика заболеваний наружных половых органов у мужчин / А.Р. Зубарев, М.Д. Митькова, М.В. Корякин, В.В. Митьков // Москва. - 1999.

47. Курбатов, Д.Г. Возможности терапии генитальной нейропатии ингибиторами фосфодиэстеразы 5 типа у больных сахарным диабетом / Д.Г. Курбатов, Р.В. Роживанов // Урология. - 2009. - №5. - С.48-49.

48. Рафальский, В.В. Подходы к рациональному выбору ингибиторов фосфодиэстеразы 5 типа / В.В. Рафальский // Фарматека. - 2004. - №19(20). - C.1-8.

49. Vardenafil Diabetes Study Group. Vardenafil, a new phosphodiesterase type 5 inhibitor, in the treatment of erectile dysfunction in men with diabetes: a mul- ticenter, double-blind, placebo-controlled, fixed-dose study / I. Goldstein, J.M. Young, J. Fischer et al. // Diabetes Care. - 2003. - Vol.26. - P.777-783.

50. Impact of diabetes mellitus on the severity of erectile dysfunction and response to treatment: analysis of data from tadalafil clinical trials / V. Fonseca, A. Seftel, J. Denne, P. Fredlund // Diabetologia. - 2004. - Vol.47. - P.1914-1923.

51. Giuliano, F. Tadalafil: a novel treatment for erectile dysfunction / F. Giuliano, L. Varfnese // Eur. Heart. J. Suppl. - 2002. - Vol.4(sup.H) - P.24-31.

52. Effects of Tadalafil on erectile dysfunction in men with diabetes / I. Sáenz de Tejada, G. Anglin, J.R. Knight, J.T. Emmick // Diabet. Care. - 2002. Vol.25. - P.2159-2164.

53. Combination therapy of Tadalafil \&Testosterone in hypogonadal nonresponders / A. Yassin, H.E. Diede, F. Saad, A. Traish // Int. J. Impot. Res. 2003. - Vol.15(Sup.6). - P.27.

54. Роживанов, Р.В. Особенности терапии эректильной дисфункции у пациентов с гипогонадизмом / Р.В. Роживанов, Д.Г. Курбатов // Врач. 2009. - №3. - С.65-69.

55. Особенности коррекции сексуальной дисфункции у мужчин, больных сахарным диабетом / Р.В Роживанов, А.Е. Лепетухин, С.А. Дубский, Д.Г. Курбатов // Сахарный диабет. 2010. - №3. - C.30-31.

56. Hackett, G. PDE5 inhibitors in diabetic peripheral neuropathy / G. Hackett 
// Int J Clin Pract. - 2006. - Vol.60. 58. Alpha lipoic acid: a new treatment P.1123-1126. forneuropathic pain in patients with di57. Ziegler, D. Clinical aspects, diag- abetes? / G.S. Mijnhout, A. Alkhalaf, nosis and therapy of diabetic neuropathy N. Kleefstra, H.J.G. Bilo // The Nether/ D. Ziegler // Ther Umsch. - 1996. - lands Journal of Medicine. - 2010. Vol.53(12). - P.948-957. Vol.64. - P.158-162. 